\title{
Episodic Activity in Active Galactic Nuclei
}

\section{J. Saikia*}

NCRA, TIFR, Pune, India

E-mail: djsencra.tifr.res.in

\section{Jamrozy}

Uniwersytet Jagielloński, Kraków, Poland

E-mail: jamrozy@oa.uj.edu.p]

\section{Konar}

ASIAA, Taiwan

E-mail: chiranjib.konar@gmail.com

\section{S. Nandi}

ARIES, Nainital

E-mail: sumana1981@gmail.com

There is increasing evidence to suggest that AGN activity may be episodic, with a wide range of possible time scales. Radio galaxies exhibit the most striking examples of episodic activity, with two or three distinct pairs of lobes on opposite sides of the active nucleus. Radio emission from earlier cycles of activity are expected to have steep radio spectra due to radiative losses, and hence be detected more easily at low radio frequencies. Inverse-Compton scattered cosmic microwave background radiation could in prinicple probe even lower Lorentz-factor particles, revealing an older population. We illustrate the time scales of episodic activity by considering different radio galaxies, discuss the possiblity of episodic activity in cluster radio sources, and a possible trend for a high incidence of $\mathrm{HI}$ absorption in sources with evidence of episodic activity.

25th Texas Symposium on Relativistic Astrophysics - TEXAS 2010

December 06-10, 2010

Heidelberg, Germany

\footnotetext{
* Speaker.
} 


\section{Introduction}

It is generally believed that the energy source of active galactic nuclei (AGN) is accretion of matter onto a supermassive black hole (SMBH) with masses ranging from $\sim 10^{6}$ to $10^{10} \mathrm{M}_{\odot}$ [1]. In this paradigm, periodic 'feeding' of the SMBH could lead to episodic AGN activity. Alternative models for intermittency are related to successful jet formation due to collimation by MHD outflows from the accretion disks [2, 3]. Marconi et al. [ 4 ] consider a scenario is which the black holes in the local Universe have grown by mass accretion during AGN phases with the average life time of these phases ranging from $\sim 1.5 \times 10^{8}$ to $10^{9} \mathrm{yr}$. A more extended review of recurrent activity in AGN can be found in Saikia \& Jamrozy [5].

Amongst galaxies harbouring an AGN, $\sim 10$ per cent of objects are radio loud, and there have been suggestions that this too may be episodic. For example, the possibility that the essential difference between a radio-loud and a radio-quiet quasar may be the epoch at which it is being observed has been suggested [6]. For the radio loud objects with extended lobes of emission on opposite sides of the nucleus, the structure and spectra of the lobes contain an imprint of the history of the source, such as interaction with the environment, radiative losses, reacceleration of particles and episodic activity of the AGN. One of the very striking examples of episodic activity in a radio galaxy is when a new pair of radio lobes is seen in addition to the outer lobes from an earlier cycle of activity. Although these have been christened more recently as 'double-double' radio galaxies (DDRGs) [7], examples of such galaxies have been reported earlier [8, 9]. An example of a tripledouble radio galaxy has also been reported by Brocksopp et al. [42].

An interesting way of probing relic emission is via X-ray observations of inverse-Comptonscattered Cosmic Microwave Background (ICCMB) radiation, which could probe lower Lorentzfactor particles and hence an older population [45]. Interesting cases of relic emission identified from X-ray observations include the radio galaxies Cygnus A [10] 11], 3C294 [12] and 4C23.56 [46].

Deep X-ray and low-frequency radio imaging studies of groups and clusters of galaxies have also revealed evidence of sources with possibly two or three cycles of activity. An interesting example is NGC5044 where Chandra observations reveal that the group hosts several radio-quiet cavities, filaments and a semi-circular cold front. The radio observations reveal possible evidence of multiple cycles of activity [13, 14].

\section{Examples of DDRGs}

As an example, the radio image of the DDRG J0041+3224 discovered using the Giant Metrewave Radio Telescope by Saikia, Konar \& Kulkarni 2006 [15] is shown in Figure 1. More than about a dozen good examples of such DDRGs are known. The size of the inner double could be small as $\sim 14 \mathrm{pc}$ as in the giga-Hertz peaked spectrum (GPS) core of J1247+6723 to several hundred kpc as in J1835+6024 [9]. The size of the outer doubles is usually large, often over a Mpc, although the highly misaligned DDRG 3C293 has an overall size of only 190 kpc [16] and the possibility that the GPS object CTA21 with an overall linear size of $\sim 0.3 \mathrm{kpc}$ might exhibit evidence of episodic activity has been explored [17]. However, the number of sources with unambiguous evidence of episodic activity is still small, and are often associated with large radio 


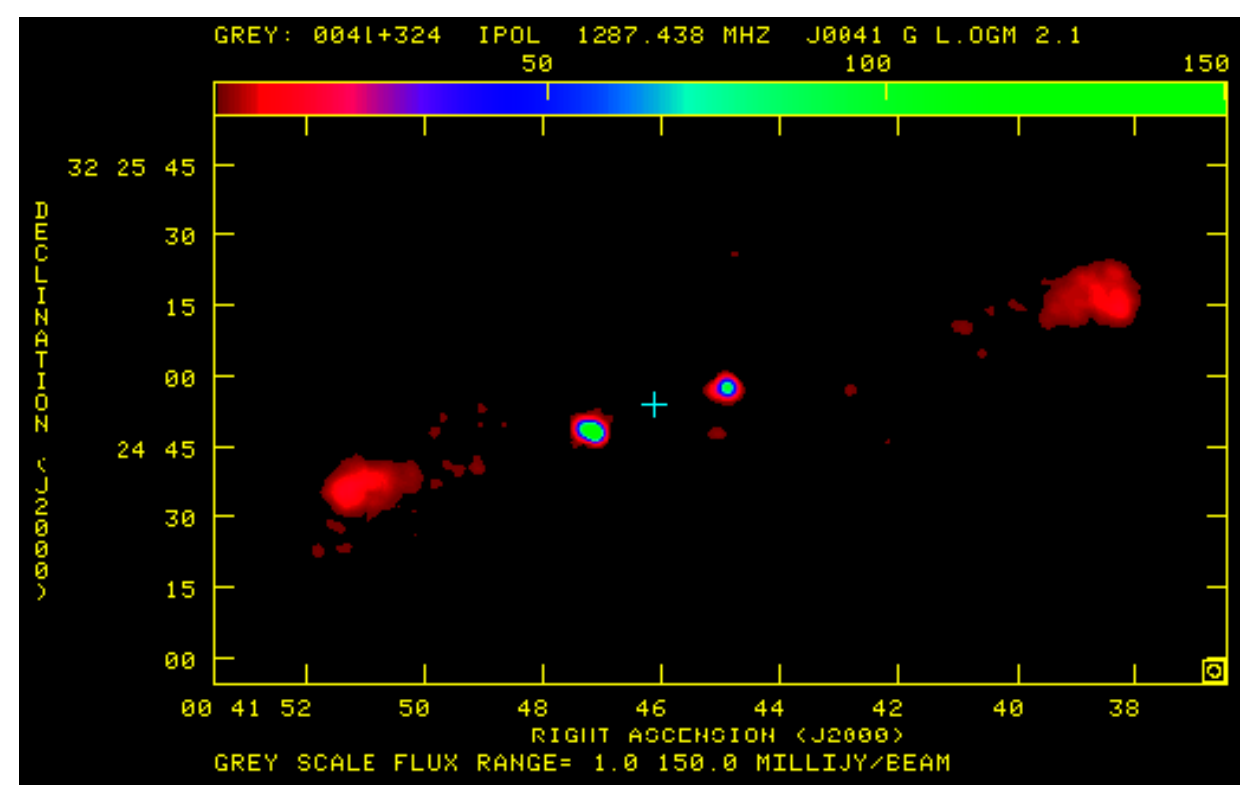

Figure 1: GMRT image of the DDRG J0041+3224 discovered using the Giant Metrewave Radio Telescope (GMRT) [15].

galaxies. This is consistent with the study by Sirothia et al. [18] where for a sample of 374 sources with a median angular size less than about 10 arcsec, there is no clear example of a DDRG.

However, there are also selection effects in trying to identify these sources. The unambiguous examples of DDRGs tend to have an edge-brightened structure for the inner double, so that these are distinguishable from knots in the jet or peaks of emission in the lobe caused by re-acceleration of particles in the lobes. The formation of the inner hotspots has been explored recently by Brocksopp et al. [43]. For these sources as well, if the time scale of jet interruption is smaller than the time required for the jet channel to collapse, the jets may not form distinct pairs of lobes. For an FRI lowluminosity radio galaxy with diffuse lobes of emission, a possible way of distingishing different cycles of activity could be from spectral-index distributions. A sharp gradient in spectral index may help demarcate the steeper-spectrum emission from an earlier cycle of activity from the more recent emission with a flatter spectrum. The radio structures may also appear to be different with the lowfrequency images detecting more extended emission from an earlier cycle of activity. However one has to be careful that these differences are not caused by differences in the resolution or coverage of the uv plane. Also, the low-luminosity radio sources are often in clusters of galaxies, and regions of flatter spectral indices may also be caused by turbulence and re-acceleration of particles as the radio plasma interacts with the intra-cluster medium. Nevertheless, possible examples of tailed FRI radio galaxies with evidence of recurrent activity have been identified from radio and X-ray studies of clusters of galaxies using spectral and structural information. One of the earlier examples (A2372) was reported by Giacintucci et al. [19], while a few more possible examples have been reported recently by Giacintucci et al. [20]. In the case of A2372 Giacintucci et al. found a significant emission gap between the inner jets coincident with the optical galaxy and the edge of 

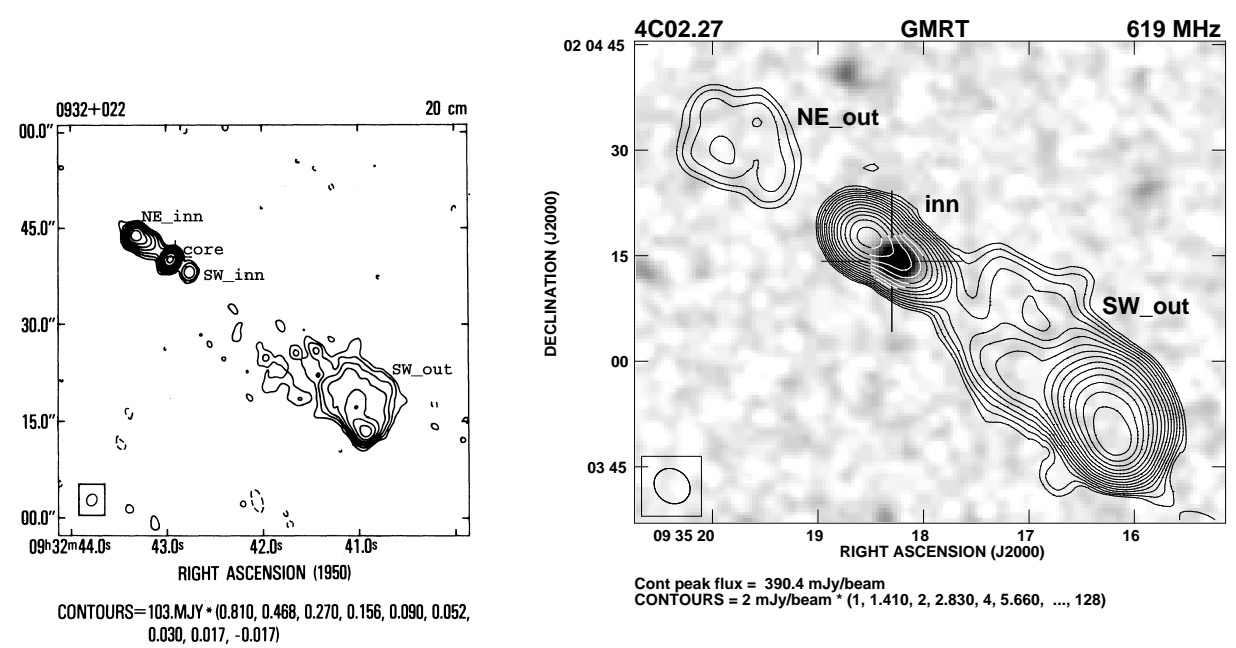

Figure 2: A Very Large Array image of the double-double radio quasar 4C02.27 at $1413 \mathrm{MHz}$ [39] on the left panel and a GMRT image at $619 \mathrm{MHz}$ [23] showing the diffuse outer lobe on the eastern side.

the outer lobes, which they interpreted to be due to recurrent activity. An example of an FRI source without distinct hot spots, but where at least two cycles of AGN activity are well accepted from radio structural information is Cen A [21]. In the large radio galaxy Her A, Gizani \& Leahy [22] have found a somewhat sharp bounday in the spectral index distribution in the lobes, which could be interpreted to be due to different cycles of activity.

If the DDRGs are associated with only galaxies, it would not be consistent with the unified scheme where the radio galaxies and quasars are believed to be intrinsically similar, but differing only in orientation. Since all the reported DDRGs were associated with galaxies, we tried to identify DDRGs associated with quasars. From a systematic comparison of FIRST and NVSS images of a large number of radio selected quasars, and imaging with the GMRT, one of the most promising examples of episodic activity in a quasar is 4C02.27 (Figure 2). Diffuse radio emission, possibly from an earlier cycle, has been imaged with the GMRT, and shown to have a steep spectral index of $\sim 1.2$ between 610 and $1400 \mathrm{MHz}$, while that of the western lobe is $\sim 1.0$ [23],. There is a prominent hot-spot in the western lobe, constraining the time scale of episodic activity to be $\sim 1.8-3.9$ Myr, depending on the orientation of the source [23]. Considering the relatively small number of giant radio quasars [28], evidence of episodic activity in 4C02.27 with an overall projected linear size of $\sim 470 \mathrm{kpc}$ is consistent with the unified scheme for radio galaxies and quasars [44].

\section{Time scales}

For a sample of 5 DDRGs, Kaiser, Schoenmakers \& Röttgering [24] find the difference in dynamical ages between the outer and inner doubles to be $\approx 10^{8} \mathrm{yr}$. Spectral ageing studies of the DDRGs J0840+2949 [25], J1453+3308 [26] and J1548-3216 [27], suggest the difference in spectral ages between the inner and outer pairs to range from $5 \times 10^{7}$ to $10^{8} \mathrm{yr}$. However, many of these sources are over a Mpc, belonging to the class of giant radio sources (e.g. [28]). Considering 

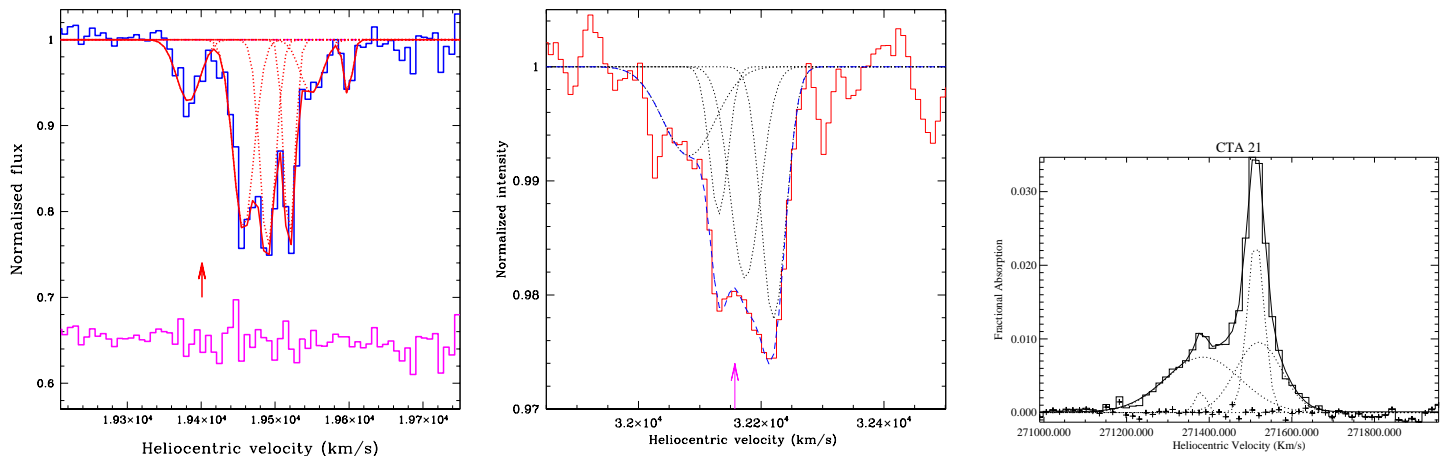

Figure 3: HI absorption spectra towards 4C29.30 (left panel [37], J1247+6723 (middle panel [31]), both obtained with the GMRT, and towards CTA21 (right panel [17]) obtained with the Arecibo telescope.

smaller sources, the total projected linear size of the inner double of of the highly misaligned DDRG 3C293 $(\mathrm{J} 1352+316)$ is $\sim 4.2 \mathrm{kpc}$, while that of the outer double is $190 \mathrm{kpc}$. The spectral ages of the inner and outer doubles, and the existence of a hot-spot suggest that the time scale of interruption of jet activity is less than $\sim 0.1 \mathrm{Myr}[16]$. In the case of Cygnus $\mathrm{A}$, with an overall linear size of $136 \mathrm{kpc}$, the time scale of interruption of jet activity has been estimated to be $\approx 10^{6}$ yr. The compact radio source, CTA21, whose radio structure has been suggested to show evidence of recurrent activity, the time scale could be in the range of $10^{4}-10^{5} \mathrm{yr}$ [40]. The different time scales are being probed using sources of different sizes.

\section{Recurrent activity and the supply of gas}

It is often speculated that the AGN activity may be triggered by a fresh supply of gas, possibly due to interactions with a companion. To test this scenario, there have been a number of searches for molecular and atomic gas in the central regions of the rejuvenated radio galaxies. Saripalli \& Mack [29] observed a sample of 9 radio galaxies with evidence of recurrent activity with the IRAM $30-\mathrm{m}$ telescope to detect CO. The only detection was 3C293, which was the only FRII radio galaxy known to have $\mathrm{CO}$ in an earlier survey of IRAS flux-density limited survey of radio galaxies [30].

Saikia, Gupta \& Konar [31] reported the detection of HI absorption towards the inner VLBIscale double of the DDRG J1247+6723, and noted the high incidence of HI absorption in sources with evidence of recurrent activity. Other examples include 3C236 [32, 33], 3C293 [34, 35], Cyg A [34], 4C29.30 [37], Cen A [21] and CTA21 [17]. Although the number of sources is small, the detection of $\mathrm{HI}$ absorption appears to be more frequent than for compact steep-spectrum and GPS sources [38]. The line profiles of HI absorption towards the cores often tend to be complex with multiple components. It would be interesting to observe these sources in HI with VLBI-scale resolution to probe the distribution and properties of the absorbing gas.

\section{Concluding remarks}

We have shown examples of recurrent AGN activity in radio galaxies and quasars inferred from 
radio and X-ray observations of radio sources in relatively low-density environments to those in clusters of galaxies. In addition to radio galaxies and quasars, it is also important to systematically probe for evidence of recurrent activity in other classes of AGN, such as for example the Seyfert galaxies. The time scales of jet interruption inferred from the present studies of radio galaxies range from $10^{5}$ to $10^{8} \mathrm{yr}$, and models would have to explain the large range of time scales. It is also possible that different models may be applicable to explain episodic activity on different time scales. Early trends appear to indicate that the radio galaxies with evidence of recurrent activity tend to exhibit $\mathrm{HI}$ in absorption with column densities in the range $\sim 8-50 \times 10^{20} \mathrm{~cm}^{-2}$ and often with complex line profiles. It would also be interesting to explore possible relationships between the coupling of episodic star formation and the rejuvenation of AGN activity, both of which may be intimately related to the supply of gas. Such studies for the giant radio galaxy 3C236 suggest that the AGN fuel supply may have been interrupted for $\sim 10^{7} \mathrm{yr}$ due to a minor merger event and has now been restored [41]. Deeper multi-wavelength studies are likely to give us new insights towards understanding recurrent AGN activity, and its effects on feedback processes and galaxy evolution.

\section{References}

[1] Rees M.J., 1984, ARA\&A, 22, 471

[2] Begelman M.C., Li Z.Y., 1994, ApJ. 426, 259

[3] Sikora M., Stawarz Ł., Lasota J.P., 2007, ApJ, 658, 815

[4] Marconi A., Risaliti G., Gilli R., Hunt L.K., Maiolino R., Salvati M., 2004, MNRAS, 351, 169

[5] Saikia D.J., Jamrozy M., 2009, BASI, 37, 63

[6] Nipoti C., Blundell K.M., Binney J., 2005, MNRAS, 361, 633

[7] Schoenmakers A.P., de Bruyn A.G., Röttgering H.J.A., van der Laan H., Kaiser C.R., 2000, MNRAS, 315,371

[8] Subrahmanyan R., Saripalli L., Hunstead R.W., 1996, MNRAS, 279, 257

[9] Lara L., Márquez I., Cotton W.D., Feretti L., Giovannini G., Marcaide J.M., Venturi T., 1999, A\&A, 348,699

[10] Steenbrugge K.C., Blundell K.M., Duffy P., 2008, MNRAS, 388, 1465

[11] Steenbrugge K.C., Heywood I., Blundell K.M., 2010, MNRAS, 401, 67

[12] Erlund M.C., Fabian A.C., Blundell K.M., Celotti A., Crawford C.S., 2006, MNRAS, 371, 29

[13] David L.P., Jones C., Forman W., Nulsen P., Vrtilek J., O’Sullivan E., Giacintucci S., Raychaudhury S., 2009, ApJ, 705, 624

[14] Giacintucci S., Vrtilek J.M., O’Sullivan E., Raychaudhury S., David L.P., Venturi T., Athreya R., Gitti M., 2009, in The Monster's Fiery Breath: Feedback in Galaxies, Groups, and Clusters

[15] Saikia D.J., Konar C., Kulkarni V.K., 2006, MNRAS, 366, 1391

[16] Joshi S.A., Nandi S., Saikia D.J., Ishwara-Chandra C.H., Konar C., 2011, MNRAS, in press

[17] Salter C.J., Saikia D.J., Minchin R., Ghosh T., Chandola Y., 2010, ApJL, 715, 117

[18] Sirothia S.K., Saikia D.J., Ishwara-Chandra C.H., Kantharia N.G., 2009, MNRAS, 392, 1403 
[19] Giacintucci S., Venturi T., Murgia M., Dallacasa D., Athreya R., Bardelli S., Mazzotta P., Saikia D.J., 2007, A\&A, 476, 99

[20] Giacintucci S., et al., 2011, ApJS, submitted (2011arXiv1103.1364G)

[21] Morganti R., Oosterloo T., Struve C., Saripalli L., 2008, A\&A, 485, L5

[22] Gizani N.A.B., Leahy J.P., 2003, MNRAS, 342, 39

[23] Jamrozy M., Saikia D.J., Konar C., 2009, MNRAS, 399, L141

[24] Kaiser C.R., Schoenmakers A.P., Röttgering H.J.A., 2000, MNRAS, 315, 38

[25] Jamrozy M., Konar C., Saikia D.J., Stawarz Ł., Mack K.-H., Siemiginowska A., 2007, MNRAS, 378, 581

[26] Konar C., Saikia D.J., Jamrozy M., Machalski J., 2006, MNRAS, 372, 693

[27] Machalski J., Jamrozy M., Konar C., 2010, A\&A, 510, 84

[28] Ishwara-Chandra C.H., Saikia D.J., 1999, MNRAS, 309, 100

[29] Saripalli L., Mack K.-H., 2007, MNRAS, 376, 1385

[30] Evans A.S., Mazzarella J.M., Surace J.A., Frayer D.T., Iwasawa K., Sanders D.B., 2005, ApJS, 159, 197

[31] Saikia D.J., Gupta N., Konar C., 2007, MNRAS, 375, L31

[32] Schilizzi R.T. et al., 2001, A\&A, 368, 398

[33] Conway J.E., Schilizzi R.T., 2000, in Conway J.E., Polatidis A.G., Booth R.S., Pihlström Y.M., eds, EVN Symp. 2000, Onsala Space Observatory, p. 123

[34] Conway J.E., 1998, ASPC, 144, 231

[35] Beswick R.J., Peck A.B., Taylor G.B., Giovannini G., 2004, MNRAS, 352, 49

[36] Emonts B.H.C., Morganti R., Tadhunter C.N., Oosterloo T.A., Holt J., van der Hulst J.M., 2005, MNRAS, 362, 931

[37] Chandola Y, Saikia D.J., Gupta N., 2010, MNRAS, 403, 269 (arXiv:0910.4427)

[38] Gupta N., Salter C.J., Saikia D.J., Ghosh T., Jeyakumar S., 2006, MNRAS, 373, 972

[39] Hintzen P., Ulvestad J., Owen F., 1983, AJ, 88, 709

[40] Reynolds C.S., Begelman M.C., 1997, ApJ, 487, L135

[41] Tremblay G.R., O’Dea C.P., Baum S.A., Koekemoer A.M., Sparks W.B., de Bruyn G., Schoenmakers A.P., 2010, ApJ, 715, 172

[42] Brocksopp C., Kaiser C.R., Schoenmakers A.P., de Bruyn A.G., 2007, MNRAS, 382, 1019

[43] Brocksopp C., Kaiser C.R., Schoenmakers A.P., de Bruyn A.G., 2011, MNRAS, 410, 484

[44] Barthel P.D., 1989, ApJ, 336, 606

[45] Mocz P., Fabian A.C., Blundell K.M., 2011, MNRAS, in press

[46] Blundell K.M., Fabian A.C., 2011, MNRAS, 412, 705 